\title{
Unusual cause of low abdominal pain
}

\author{
F. Gelders ${ }^{1}$, J. Vandewalle', M. Struyve ${ }^{1,2}$ \\ (1) Department of Gastroenterology and Hepatology, University Hospitals Leuven, KU Leuven, Leuven; (2) Department of Gastroenterology and Hepatology, Ziekenhuis \\ Oost-Limburg (ZOL), Genk, Belgium.
}

\section{Question}

A 53-year-old woman, G2P2, was referred to the outpatient clinic because of chronic abdominal pain. Abdominal examination revealed tenderness in the right lower quadrant without peritoneal signs and blood analysis was normal. To exclude slow transit constipation a pellet study was performed: plain abdominal radiography showed the presence of all ingested pellets and an abnormal, slightly lateralized and angulated position of the intrauterine device, a levonorgestrel intrauterine system called Mirena ${ }^{\circledR}$ which was placed five years earlier (figure 1). Subsequently an abdominal computed tomography was executed. These images are shown in figure 2 .

What is the diagnosis?

\section{Answer}

The CT-images revealed a migration of the intrauterine device (IUD) with one side arm perforating the sigmoid colon while the other arm was embedded in the mesosigmoidal fat.

Colon perforation due to IUD migration is a very rare but possible serious complication of IUD insertion, with the sigmoid colon being most commonly affected $(1,2)$. IUD-mediated uterine perforations mostly have a silent or low-grade course, although more severe signs such as peritonitis, bleeding or intestinal obstruction are possible due to perforation of adjacent organs $(2,3)$. However it is postulated that one-third of patients with an intestinal perforation experience mild or even no symptoms, as in our patient (2,3). In nearly all cases laparoscopic retrieval is necessary. Endoscopic removal can only be considered in well-selected patients with a silent IUD migration into the digestive lumen or the innermost portion of the colonic wall, when withdrawal can be carried out under direct vision of the entire IUD to prevent injury of adjacent structures (3).

After successful laparoscopic removal with closure of the perforated sigmoid colon wall, her complaints quickly disappeared without administration of antibiotics.

\section{Potential competing interests}

None.

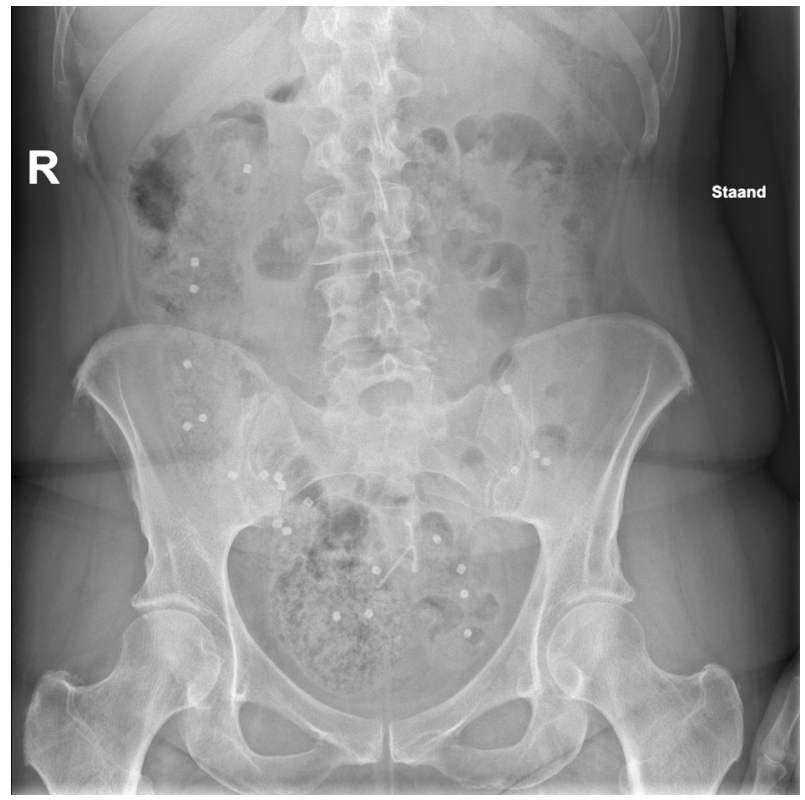

Fig. 1. - Plain abdominal radiography showing an abnormal, slightly lateralized and angulated position of the IUD.

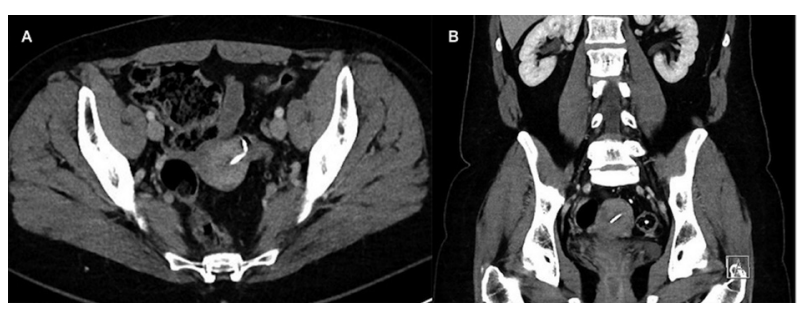

Fig 2. - (A) (B) Abdominal CT with migration of the IUD through the left lateral uterine wall perforating the sigmoid colon.

\section{References}

1. ROWLANDS S., OLOTO E., HORWELL D. Intrauterine devices and risk of uterine perforation: current perspectives. Open access Journal of Contraception, 2016; $7:$ 19-32.

2. CARMODY K, SCHWARTZ B., CHANG A. Extrauterine Migration of a Mirena Intrauterine Device. The Journal of Emergency Medicine, 2011; 4(2): 161-165.

3. ARSLAN A, KANAT-PEKTAS M., YESILYURT H., BILGE U. Colon penetration by a copper intrauterine device: a case report with literature review. Arch. Gynecol. Obstet., 2009; 279: 395-7.

Correspondence to: France Gelders, Refugehof 9/0001, 3000 Leuven.

E-mail: france.gelders@student.kuleuven.be

Submission date : 08/02/2021

Acceptance date : 15/03/2021 